\title{
On the Nonlinear Stability of Inviscid Homogeneous Shear Flows in Sea Straits of Arbitrary Cross Sections
}

\author{
V Ramakrishnareddy* and M Subbiah ${ }^{\dagger}$
}

\begin{abstract}
In this paper we study the nonlinear stability of steady flows of inviscid homogeneous fluids in sea straits of arbitrary cross sections. We use the method of Arnol'd [1] to obtain two general stability theorems for steady basic flows with respect to finite amplitude disturbances. For the special case of plane parallel shear flows we find a finite amplitude extension of the linear stability result of Deng et al [2]. We also present some examples of basic flows which are stable to finite amplitude disturbances.
\end{abstract}

PACS: 47.20

Keywords: Nonlinear stability, inviscid shear flows, variable bottom, sea straits.

\section{Introduction}

The study of linear stability of shear flows in sea straits with variable cross sections was initiated by Pratt et al [3]. It was found in Pratt et al [3] that the resultant stability problem is an extended version of the well known Taylor-Goldstein problem of Hydrodynamic Stability. In Deng et al [2] a systematic derivation of the stability equation, namely, the extended Taylor-Goldstein equation was derived and many general analytical results were

\footnotetext{
* Department of Mathematics, Pondicherry University, Pondicherry605014 , India

† Department of Mathematics, Pondicherry University, Pondicherry605014, India
} 
obtained for this problem. It was found that these results do not depend on the topography and hence the role of topography on the stability of stratified shear flows in sea straits is not revealed by any of these results. However two examples of basic flows were shown to be linearly unstable by numerical computations in Pratt et al [3]. In one example the topography was found to decrease the growth rate though the basic flow remains unstable.

In Deng et al [2] the special case of homogeneous shear flows was also considered in their stability analysis. The stability problem in this case turns out to be an extended version of the well known Rayleigh problem of Hydrodynamic Stability. For this problem Deng et al [2] found that a necessary condition for instability is that $\left(\frac{U_{0}{ }^{\prime}}{b}\right)^{\prime}$ should change sign at least once in the flow domain where $U_{0}$ is the basic flow velocity, $b(z)$ is the width function of the channel and a prime denotes differentiation with respect to the vertical coordinate variable $z$. If $T=\frac{b^{\prime}(z)}{b(z)}$ represents the topography variable the above result can be stated as follows: A necessary condition for instability is that $U_{0} "-T U_{0}$ 'should change sign at least once in the flow domain. In other words the basic flow is stable if $U_{0}{ }^{\prime}-T U_{0}{ }^{\prime}>0$ or $U_{0}{ }^{\prime \prime}-T U_{0}{ }^{\prime}<0$ throughout the flow domain. It is interesting to note that the topography $T(z)$ appears in this important result. Consequently $U_{0}{ }^{\prime \prime}-T U_{0}{ }^{\prime}$ should become zero somewhere in the flow domain and let $z=z_{s}$ be a point in the flow domain such that $\left(U_{0}{ }^{\prime}-T U_{0}{ }^{\prime}\right)\left(z_{s}\right)=0$. If $U_{s}=U_{0}\left(z_{s}\right)$ then an extension of the above result is that a necessary condition for instability is that $\left(U^{\prime \prime}{ }_{0}-T U_{0}{ }^{\prime}\right)\left(U_{0}-U_{s}\right)<0$ at least once in the flow domain. It may be noted here that the above results are extensions of the Rayleigh's theorem and the Fjortoft's theorem, respectively, of the Rayleigh problem of hydrodynamic stability [cf: Drazin and Reid [4]]. Though the above results depend on the topography it is difficult to see any tendency towards stability or instability due to topographic variations. To see this point Deng et al [2] considered the special case of 
$T=T_{0}=$ a constant $>0$. Then the extended Rayleigh theorem gives $\frac{U_{0}^{\prime \prime}}{U_{0}^{\prime}}=T_{0}$ at least once in the flow domain. If $\frac{U_{0}^{\prime \prime}}{U_{0}^{\prime}}$ is bounded above in the flow domain it implies that the flow is stable when $T_{0}$ is sufficiently large and if $\frac{U_{0}^{\prime \prime}}{U_{0}^{\prime}}$ is bounded below by a positive constant in the flow domain then a sufficiently small $T_{0}$ will make the flow a stable one. Thus we can see that some flows which are unstable in the absence of topography $\left(T_{0}=0\right)$ may become stable in the presence of the topography $T_{0}$. Now consider the flow $U_{0}=\frac{z^{2}}{2}$. Since $U_{0} "=1$ it follows that this flow is stable in the absence of topography $\left(T_{0}=0\right)$. However $U_{0}{ }^{\prime}-T U_{0}{ }^{\prime}=1-T_{0}(z)$ and it becomes zero at $z_{s}=\frac{1}{T_{0}}$, and hence the necessary condition for instability is satisfied. So if this flow is unstable then it is only due to the topography $T_{0}>0$.Further analytical results on this problem has been obtained in Subbiah and Ganesh [5] and Subbiah and Ganesh [6]. In particular if $T=T_{0}$ is a positive constant a short wave stability result has been obtained in Subbiah and Ganesh [6]. Also the role of the Reynolds stress on this problem has been discussed in Subbiah and Ramakrishnareddy [7].

All the above mentioned results on the stability of stratified or homogeneous shear flows are restricted to infinitesimal normal mode disturbances only and the mathematical analysis is linear. However to understand the stability of shear flows in sea straits with respect to finite amplitude disturbances it is necessary to develop a nonlinear stability analysis. In the present paper we consider the nonlinear stability of inviscid homogeneous shear flows in sea straits of arbitrary cross sections by using the method of Arnol'd [1] developed in the context of flows without topography. The nonlinear stability of viscous fluids is studied by the method of Serrin (see, for example, Joseph [8]).But this method, also known as the energy method of hydrodynamic stability does 
not apply to inviscid fluids. Consequently, a variational method was developed by Arnol'd [9] for the nonlinear stability analysis of inviscid incompressible fluids. As this method was not giving rigorous stability theorems a new method of nonlinear stability analysis was developed in Arnol'd [1].This method, also known as the second method of Arnol'd, has been used extensively in the nonlinear stability analysis of many geophysical fluid flows (see, for example, Arnold and Khesin [10], Marchioro and Pulvirenti [11] and Holm et al [12]).

Now we shall present the results of this paper. In Section 2 we formulate the problem by deriving the nonlinear partial differential equation governing the motion of an inviscid incompressible homogeneous fluid in a channel with variable bottom and the associated boundary conditions. In Section 3 we obtain a conserved quantity of this problem which is later used in the proof of the nonlinear stability results. The two nonlinear stability theorems proved in the present paper are the following: If $\Psi(x, z)$ is the stream function and $Q(x, z)$ is the vorticity divided by the width function of a steady flow then it is nonlinearly stable to finite amplitude perturbations having zero circulation around the boundary of the domain whenever (i) $\Psi^{\prime}(Q)>0$ or (ii) $\Psi^{\prime}(Q)<0$ and the domain is sufficiently small, in a sense specified later. An interesting corollary of the first theorem is the following: For the special case of plane parallel flows $\Psi=\Psi(z)$ and $Q=Q(z)$ and if $Q(z)$ is monotonic, that is, if $Q^{\prime}(z)>0$ or $Q^{\prime}(z)<0$ throughout the flow domain, then that flow is nonlinearly stable. In the absence of topography this result was obtained by McIntyre and Shepherd [13].In terms of the basic velocity $U_{0}(z)$ and topography $T(z)$ this means that the basic plane parallel flow is nonlinearly stable if $U_{0}{ }^{\prime}-T U_{0}{ }^{\prime}>0$ or $U_{0}{ }^{\prime}-T U_{0}{ }^{\prime}<0$ throughout the flow domain. Thus it is clear that the sufficient conditions for linear stability with respect to infinitesimal normal mode disturbances obtained by Deng et al [2] are also sufficient conditions for nonlinear stability with respect to finite amplitude disturbances. We have also presented some examples of basic flows which are stable by the above general results. In particular an exchange flow with velocity 
$U_{0}(z)=\tanh \left(z-\frac{1}{2}\right)$ is shown to be nonlinearly stable by our second stability theorem where the topography is given by $T(z)=\tanh \left(z-\frac{1}{2}\right)$ and the depth of the fluid layer $D$ is small, i.e., $D$ is less than $D_{0} \approx 1$.3. As Pratt et al [3] have observed exchange flows in the sea strait Bab al Mandab our results are expected to facilitate the understanding of the dynamics of shear flows in sea straits.

\section{Formulation of the Problem}

In Deng et al [2] the problem of linear stability of shear flows of inviscid incompressible homogeneous as well as stratified fluids in sea straits of arbitrary cross section has been formulated. In the present paper we consider only the special case of a homogeneous fluid but consider the nonlinear stability of such flows. If $(u, v, w)$ is the velocity, $\rho$ is the density which is a constant for a homogeneous fluid and $p$ is the pressure of the fluid then the motion is governed by the Euler equations of fluid dynamics. But in Deng et al [2] a special class of fluid flows satisfying the condition $\frac{\partial}{\partial y}(u, w, \rho, p)=0$ was considered. That is, these flow variables $u, w, \rho$ and $p$ can vary with the elevation $z$ and with the along channel direction $x$, but not with the cross channel direction $y$.However the cross channel component $v$ of the velocity can vary with cross channel direction $y$.For such flows of homogeneous fluid the governing equations are

$$
\begin{gathered}
\frac{d u}{d t}=-\frac{\partial p}{\partial x}, \\
\frac{d v}{d t}=0, \\
\frac{d w}{d t}=-\frac{\partial p}{\partial z},
\end{gathered}
$$




$$
\frac{\partial u}{\partial x}+\frac{\partial v}{\partial y}+\frac{\partial w}{\partial z}=0
$$

where $\frac{d}{d t}=\frac{\partial}{\partial t}+u \frac{\partial}{\partial x}+v \frac{\partial}{\partial y}+w \frac{\partial}{\partial z}$; It is seen from (2) that the velocity component in the $y$-direction is preserved and so can be ignored in the stability analysis. If the lateral walls of the domain are given by $y=y_{R}(z)$ and $y=y_{L}(z)$ then Deng et al [2] have shown that the last equation (4) becomes

$$
\frac{\partial(b u)}{\partial x}+\frac{\partial(b w)}{\partial x}=0
$$

where $b(z)=y_{R}(z)-y_{L}(z)$ is the width function of the channel. Furthermore, a stream function $\psi(x, z, t)$ can be introduced by the equations

$$
\begin{gathered}
b w=\frac{\partial \psi}{\partial x}, \\
b u=-\frac{\partial \psi}{\partial z} .
\end{gathered}
$$

Moreover in the case of homogeneous fluid whose stability is considered in this paper, Deng et al [2] have shown that

$$
\frac{D}{D t}\left(\frac{\zeta}{b}\right)=0
$$

where $\zeta=\frac{\partial w}{\partial x}-\frac{\partial u}{\partial z}$ is the scalar vorticity and $\frac{D}{D t}=\frac{\partial}{\partial t}+u \frac{\partial}{\partial x}+w \frac{\partial}{\partial z}$ is the Eulerian derivative of fluid flows in the $x-z$ plane which is different from $\frac{d}{d t}$ used earlier. This nonlinear partial differential equation was derived in Deng et al [2] and it was observed that this equation expresses the conservation of vorticity divided by the width function of a fluid column. However the stability analysis given in Deng et al [2] is limited to basic flows which are plane parallel flows and to disturbances 
which are infinitesimal normal mode disturbances. Consequently their stability analysis is based only on the linearized version of the equation (8) and not on the full nonlinear equation. The flow domain considered in this paper is given by $\Omega: 0 \leq x \leq X, 0 \leq z \leq D$, where $X$ and $D$ are positive constants. Here $X$ is length of the channel and $D$ is the depth of the channel.In terms of the stream function $\psi$ the equation (8) becomes

$$
\frac{D}{D t}\left(\frac{\frac{\partial}{\partial x}\left(\frac{1}{b} \frac{\partial \psi}{\partial x}\right)+\frac{\partial}{\partial z}\left(\frac{1}{b} \frac{\partial \psi}{\partial z}\right)}{b(z)}\right)=0
$$

i.e.,

$$
\frac{D}{D t} q=0
$$

where $q=\frac{\frac{\partial}{\partial x}\left(\frac{1}{b} \frac{\partial \psi}{\partial x}\right)+\frac{\partial}{\partial z}\left(\frac{1}{b} \frac{\partial \psi}{\partial z}\right)}{b(z)}$, is the scalar vorticity of the flow divided by the width function of the channel. From the equations (1),(3), and (4) one can show that

$$
\frac{D}{D t} \iint_{\Omega} b\left[\frac{u^{2}+w^{2}}{2}\right] d x d z=0
$$

which is a statement of the conservation of kinetic energy of the flow in the channel. In terms of the stream function this can be stated as

$$
\frac{D}{D t} \iint_{\Omega}\left[\frac{\left(\frac{\partial \psi}{\partial x}\right)^{2}+\left(\frac{\partial \psi}{\partial z}\right)^{2}}{2 b}\right] d x d z=0
$$

Equation (8) can be written in the form 


$$
\frac{\partial q}{\partial t}+\frac{1}{b(z)} J(q, \psi)=0
$$

where $J(.,$.$) is the Jacobian.$

Now we shall state the stability problem considered in this paper. Consider a steady basic flow with stream function $\Psi(x, z)$ and vorticity per width function $Q(x, z)$.From (11) it follows that $\Psi$ and $Q$ should satisfy $J(Q, \Psi)=0$ and from this we can take $\Psi=\Psi(Q)$ provided that $\nabla Q \neq 0$.If this basic flow is disturbed so that the disturbed flow variables are $\Psi+\phi(x, z, t)$ and $Q+q(x, z, t)$ then we want to prove that the basic flow $(\Psi, Q)$ is nonlinearly stable to disturbances having zero circulation around the boundary $\partial \Omega$ of $\Omega$; i.e., $\int_{\Omega} \vec{u} \cdot d \vec{s}=0$, where $\vec{u}=(u, w)$ is the velocity of the fluid. It may be noted here that if $\int_{\partial \Omega} \vec{u} \cdot d \vec{s}=0$ at $t=0$ then it is zero for all time by Kelvin's circulation theorem.

\section{Stability Results}

In this section we shall state and prove the nonlinear stability theorems for the problem under consideration. For that we need some conservation results. The first conservation result is the following

\section{Lemma 1}

$$
\frac{D}{D t} \iint_{\Omega} \frac{(\nabla \psi)^{2}}{2 b} d x d z=0
$$

This is the statement of the conservation of kinetic energy given in (10) and its proof is routine. The second conservation result is the following. 


\section{Lemma 2}

$$
\frac{D}{D t} \iint_{\Omega} b(z) \Phi(\eta) d x d z=0 .
$$

where $\eta=\frac{\zeta}{b(z)}=\frac{\frac{\partial}{\partial x}\left(\frac{1}{b} \frac{\partial \psi}{\partial x}\right)+\frac{\partial}{\partial z}\left(\frac{1}{b} \frac{\partial \psi}{\partial z}\right)}{b(z)}$ is the vorticity divided by the width function and $\Phi(\eta)$ is any smooth function of $\eta$. The proof follows from the fact that $\frac{D}{D t} \Phi(\eta)=0$ and the equation of continuity (5).

The two conserved quantities given above can be combined to get a single conserved quantity.

\section{Lemma 3}

For any flows the quantity

$$
H(\psi(x, z, t))=\iint_{\Omega}\left[\frac{(\nabla \psi)^{2}}{2 b}+b \Phi(\eta)\right] d x d z \text { is conserved. }
$$

It is clear that for the steady basic flow $(\Psi(x, z), Q(x, z))$ the quantity $H(\Psi)$ is conserved. Also it is clear that for the disturbed flow $(\Psi+\phi(x, z, t), Q+q(x, z, t))$, the quantity $H(\Psi+\phi, Q+q)$ is conserved. Consequently the difference $H(\Psi+\phi, Q+q)-H(\Psi, Q)$ is also a conserved quantity. Therefore it easily follows that the quantity

$$
\begin{aligned}
H(\phi) & =H(\psi+\phi)-H(\psi) \\
& =\iint_{\Omega}\left[\frac{(\nabla \phi)^{2}}{2 b}+b\left(\Phi(Q+q)-\Phi(Q)-\Phi^{\prime}(Q) q\right)\right] d x d z
\end{aligned}
$$

is conserved for perturbations satisfying the condition of zero circulation around the boundary $\partial \Omega$. This means the following. 


\section{Lemma 4}

$$
H(\phi(x, z, t))=H(\phi(x, z, 0)) .
$$

Now we shall present the first nonlinear stability theorem.

\section{Theorem 1 (First Stability Theorem)}

If the basic flow with stream function $\Psi$ and vorticity divided by the width function $Q$ satisfies $0<c \leq \Psi^{\prime}(Q) \leq C<\infty$, for real constants $c$ and $C$ then it is stable in the norm \|\|$_{+}$given by

$$
\|\phi\|_{+}^{2}=\iint_{\Omega}\left[\frac{(\nabla \phi)^{2}}{b}+b c q^{2}\right] d x d z .
$$

Proof:

Define $\Phi(\eta)=\int_{0}^{\eta} \Psi(s) d s$, where $Q_{\min } \leq \eta \leq Q_{\max }$.

By hypothesis of the theorem we have

$$
0<c \leq \Phi^{\prime \prime}(\eta) \leq C<\infty
$$

in the range $Q_{\min } \leq \eta \leq Q_{\max }$. We can extended $\Phi(\eta)$ to the whole real line subject to the above inequalities. We consider the extended function $\Phi(\eta)$ hereafter.

Integrating (12) over $\left(\xi, \xi+h_{1}\right)$ we get

$$
c h_{1} \leq\left[\Phi^{\prime}\left(\xi+h_{1}\right)-\Phi^{\prime}(\xi)\right] \leq C h_{1}
$$

Integrating (13) w.r.to $h_{1}$ over $(0, h)$ and multiplying by $b(z)$ we get

$$
\frac{b c h^{2}}{2} \leq b\left[\Phi(\xi+h)-\Phi(\xi)-\Phi^{\prime}(\xi) h\right] \leq \frac{b C h^{2}}{2} .
$$

So we have

$$
\frac{b c q^{2}}{2} \leq b\left[\Phi(Q+q)-\Phi(Q)-\Phi^{\prime}(Q) q\right] \leq \frac{b C q^{2}}{2} .
$$


Consequently adding $\frac{|\nabla \phi|^{2}}{2 b}$ and integrating over the flow domain $\Omega$ we have

$$
\begin{aligned}
& \iint_{\Omega}\left[\frac{|\nabla \phi|^{2}}{2 b}+\frac{b c q^{2}}{2}\right] d x d z \\
& \quad \leq \iint_{\Omega}\left[\frac{|\nabla \phi|^{2}}{2 b}+b\left[\Phi(Q+q)-\Phi(Q)-\Phi^{\prime}(Q) q\right]\right] d x d z \quad(\text { by }(14)) \\
& \quad=H(\phi)=H\left(\phi_{0}\right) \quad(\text { by Lemma } 4) \\
& \quad \leq \iint_{\Omega}\left[\frac{\left|\nabla \phi_{0}\right|^{2}}{2 b}+b\left[\Phi\left(Q+q_{0}\right)-\Phi(Q)-\Phi^{\prime}(Q) q_{0}\right]\right] d x d z \\
& \quad \leq \iint_{\Omega}\left[\frac{\left|\nabla \phi_{0}\right|^{2}}{2 b}+\frac{b C q_{0}^{2}}{2}\right] d x d z \quad(\text { by }(14)) \\
& \quad \leq \frac{C}{c} \times \frac{c}{C} \iint_{\Omega}\left[\frac{\left|\nabla \phi_{0}\right|^{2}}{2 b}+\frac{b C q_{0}^{2}}{2}\right] d x d z \\
& \quad \leq \frac{C}{c} \iint_{\Omega}\left[\frac{\left|\nabla \phi_{0}\right|^{2}}{b}+b c q_{0}^{2}\right] d x d z \\
& \quad\left\|\phi_{0}\right\|_{+}^{2} \cdot
\end{aligned}
$$

Given $\varepsilon>0 \exists \delta(\varepsilon)$ given by $\delta^{2}=\frac{c}{C} \varepsilon^{2}$ so that $\left\|\phi_{0}\right\|_{+}^{2}<\delta^{2}$ implies $\|\phi\|_{+}^{2} \leq \varepsilon^{2}$. The proof of the theorem is completed. 


\section{Example 1}

Consider the basic flow with velocity $U_{0}=e^{z^{2}}, 0 \leq z \leq D$ and $b(z)=z$ so that $T(z)=\frac{1}{z}$ and $\Psi^{\prime}(Q)=\frac{b^{2} U_{0}(z)}{U_{0}{ }^{\prime \prime}(z)-T U_{0}{ }^{\prime}(z)}>0$. It follows that this basic flow is stable by the first stability theorem for any $D>0$.

Now we shall derive two corollaries of this theorem for parallel flows.

\section{Corollary 1}

If the basic flow is such that $\Psi=\Psi(z)$ and $Q=Q(z)$ with $Q^{\prime}(z) \neq 0$, then under the conditions $0<c \leq \Psi^{\prime}(Q) \leq C<\infty$, we have the following bounds.

$$
\iint_{\Omega}\left[\frac{(\nabla \phi)^{2}}{b}+\mu b q^{2}\right] d x d z \leq \frac{\left(\frac{\Psi^{\prime}(z)}{Q^{\prime}(z)}\right)_{\max }}{\left(\frac{\Psi^{\prime}(z)}{Q^{\prime}(z)}\right)_{\min }} \int_{\Omega}\left[\frac{\left(\nabla \phi_{0}\right)^{2}}{b}+\mu b q_{0}^{2}\right] d x d z,
$$

for any $\mu$ in the range $\left(\frac{\Psi^{\prime}(z)}{Q^{\prime}(z)}\right)_{\text {min }} \leq \mu \leq\left(\frac{\Psi^{\prime}(z)}{Q^{\prime}(z)}\right)_{\max }$.

Proof:

Let $c=\left(\frac{\Psi^{\prime}(z)}{Q^{\prime}(z)}\right)_{\min }$ and $C=\left(\frac{\Psi^{\prime}(z)}{Q^{\prime}(z)}\right)_{\max }$ then under the conditions $0<c \leq \Psi^{\prime}(Q) \leq C<\infty$, we have the inequalities

$$
\iint_{\Omega}\left[\frac{1}{b}(\nabla \phi)^{2}+b c q^{2}\right] d x d z \leq \iint_{\Omega}\left[\frac{1}{b}\left(\nabla \phi_{0}\right)^{2}+\mu b q_{0}^{2}\right] d x d z
$$

is true. Therefore, for any $\mu$ in the range $c \leq \mu \leq C$, we have 


$$
\begin{aligned}
\iint_{\Omega}\left[\frac{1}{b}(\nabla \phi)^{2}+\mu b q^{2}\right] d x d z & \leq \frac{\mu}{c} \iint_{\Omega}\left[\frac{1}{b}(\nabla \phi)^{2}+b c q^{2}\right] d x d z \\
& \leq \frac{\mu}{c} \iint_{\Omega}\left[\frac{1}{b}\left(\nabla \phi_{0}\right)^{2}+b C q_{0}^{2}\right] d x d z \\
& \leq \frac{C}{c} \iint_{\Omega}\left[\frac{1}{b}\left(\nabla \phi_{0}\right)^{2}+\mu b q_{0}^{2}\right] d x d z .
\end{aligned}
$$

Hence the result is proved.

\section{Corollary 2}

If the basic flow is a parallel flow with $\Psi=\Psi(z)$ and $Q=Q(z)$ with monotonic $Q$ then under the conditions $0<c \leq \Psi^{\prime}(Q) \leq C<\infty$, we have the following bounds

$$
\iint_{\Omega} b q^{2} d x d z \leq \frac{\left|Q^{\prime}(z)\right|_{\max } b_{\max }}{\left|Q^{\prime}(z)\right|_{\min } b_{\min }} \iint_{\Omega} b q_{0}^{2} d x d z .
$$

This can be proved on the lines of the proof of the similar result in Subbiah and Padmini [14] and so the details of the proof are not given here.

\section{Example 2}

Consider the basic flow with velocity $U_{0}(z)=1-z, 0 \leq z \leq D$, and $b(z)=e^{z}$ so that $T=1$ and $Q^{\prime}(z)=\frac{-U_{0}{ }^{\prime \prime}+T U_{0}{ }^{\prime}}{b}=\frac{-1}{e^{z}}<0$ and $\Psi^{\prime}(Q)=\frac{b^{2} U_{0}(z)}{U_{0}{ }^{\prime}-T U_{0}{ }^{\prime}}=(1-z) e^{z}>0$ in $0 \leq z \leq D$ if $D<1$. This flow is stable by the corollary 2 .

For the parallel flows we have $Q=\frac{-U_{0}{ }^{\prime}}{b}$. For monotonic $Q$ we have $Q^{\prime}(z)>0$ or $Q^{\prime}(z)<0$ throughout the domain. This means that $\frac{-U_{0}{ }^{\prime \prime}(z)+T U_{0}{ }^{\prime}(z)}{b(z)}$ is positive throughout or negative 
throughout. Hence by Corollary 2 it follows that a parallel flow is nonlinearly stable if $U_{0}{ }^{\prime \prime}(z)-T U_{0}{ }^{\prime}(z)<0$ or $>0$ throughout the flow domain. Thus Corollary 2 gives the nonlinear extension of the linear stability result of Deng et al [2].

Now we shall state and prove our second nonlinear stability theorem. For that we need the following inequality which is known as the Poincare inequality.

\section{Lemma 5}

If $\psi(x, z, t)$ is a function vanishing on the boundary of the domain $\Omega: 0 \leq x \leq X, 0 \leq z \leq D$, where $X>0$ and $D>0$ are constants then

$$
\iint_{\Omega}(\Delta \psi)^{2} d x d z \geq \lambda_{1} \iint_{\Omega}(\nabla \psi)^{2} d x d z
$$

where $\lambda_{1}=\pi^{2}\left(\frac{1}{X^{2}}+\frac{1}{D^{2}}\right)$.

For a proof of this inequality and its use in stability analysis of fluid flows, one can see, for example, McIntyre and Shepherd [13]. From Lemma 5 we get the following.

\section{Lemma 6}

$$
\iint_{\Omega} b q^{2} d x d z \geq \frac{\lambda_{1}}{b^{2}{ }_{\max }} \iint_{\Omega} \frac{(\nabla \phi)^{2}}{b} d x d z
$$

where $\phi$ vanishes on the boundary of $\Omega$.

\section{Theorem 2 (Second Stability Theorem)}

If the basic flow with stream function $\Psi$ and vorticity divided by width function $Q$ satisfies $0<c \leq-\Psi^{\prime}(Q) \leq C<\infty$, then the basic flow is nonlinearly stable in the norm given by

$$
\|\phi\|_{-}^{2}=\iint_{\Omega} b q^{2} d x d z
$$

whenever the domain is sufficiently small. 
Remark: The second stability theorem gives the stability of a flow where the domain is small in the sense that $c-\frac{b^{2}{ }_{\max }}{\lambda_{1}}>0$ that is, $\pi^{2}\left(\frac{1}{X^{2}}+\frac{1}{D^{2}}\right)>\frac{b_{\max }^{2}}{c}$. This will be satisfied if $D$ is small.

Now we shall give the proof of theorem 2 .

\section{Proof:}

Instead of (12) we have $0<c \leq-\Phi "(Q) \leq C<\infty$.

Proceeding as in the proof of Theorem 1 we have

$$
\frac{b c h^{2}}{2} \leq-b\left[\Phi(\xi+h)-\Phi(\xi)-\Phi^{\prime}(\xi) h\right] \leq \frac{b C h^{2}}{2} .
$$

Hence we have

$$
\begin{aligned}
& \iint_{\Omega}\left[\frac{-(\nabla \phi)^{2}}{2 b}+\frac{b c q^{2}}{2}\right] d x d z \\
& \quad \leq \iint_{\Omega}\left[\frac{-(\nabla \phi)^{2}}{2 b}-b\left[\Phi(Q+q)-\Phi(Q)-\Phi^{\prime}(Q) q\right]\right] d x d z \\
& \quad=-H(\phi)=-H\left(\phi_{0}\right) \\
& \quad=\iint_{\Omega}\left[\frac{-\left(\nabla \phi_{0}\right)^{2}}{2 b}-b\left[\Phi\left(Q+q_{0}\right)-\Phi(Q)-\Phi^{\prime}(Q) q_{0}\right]\right] d x d z \\
& \quad \leq \iint_{\Omega}\left[\frac{-\left(\nabla \phi_{0}\right)^{2}}{2 b}+\frac{b c q_{0}^{2}}{2}\right] d x d z .
\end{aligned}
$$

Consequently we have

$$
\left.\iint_{\Omega}\left[\frac{-(\nabla \phi)^{2}}{b}+b c q_{0}^{2}\right]\right] d x d z \leq C \iint_{\Omega}\left[b q_{0}^{2}\right] d x d z
$$

Using Poincare's inequality we have 


$$
\iint_{\Omega} b q^{2} d x d z \geq \frac{\lambda_{1}}{b^{2}} \iint \frac{(\nabla \phi)^{2}}{b} d x d z
$$

Using this in (15) gives

$$
\left(c-\frac{b_{\max }^{2}}{\lambda_{1}}\right) \iint_{\Omega} b q^{2} d x d z \leq C \iint_{\Omega} b q_{0}^{2} d x d z
$$

Now, if $c-\frac{b_{\text {max }}^{2}}{\lambda_{1}}>0$, then it follows that

$$
\begin{gathered}
\iint_{\Omega} b q^{2} d x d z \leq \frac{C}{c-\frac{b_{\text {max }}^{2}}{\lambda_{1}} \iint_{\Omega} b q_{0}^{2} d x d z} \\
\text { i.e., }\|\phi\|_{-}^{2} \leq \frac{C}{c-\frac{b_{\text {max }}^{2}}{\lambda_{1}}}\left\|\phi_{0}\right\|_{-}^{2}
\end{gathered}
$$

Given $\varepsilon>0 \exists \delta(\varepsilon)>0$ given by $\delta^{2}=\frac{c-\frac{b_{\text {max }}^{2}}{\lambda_{1}}}{C} \varepsilon^{2}$ so that the basic flow is stable in the norm \|\|$_{-}$.

\section{Example 3}

Now we shall consider the exchange flow with velocity $U_{0}(z)=\tanh \left(z-\frac{1}{2}\right)$ and width function $b(z)=\cosh \left(z-\frac{1}{2}\right)$, where $0 \leq z \leq D$

Then $T(z)=\tanh \left(z-\frac{1}{2}\right), \quad$ and $\quad \Psi^{\prime}(Q)=\frac{-\cosh ^{4}\left(z-\frac{1}{2}\right)}{3}<0$, $c=\left(-\Psi^{\prime}(Q)\right)_{\min }=\frac{1}{3}, \quad C=\left(-\Psi^{\prime}(Q)\right)_{\max }=\frac{\cosh ^{4}\left(D-\frac{1}{2}\right)}{3} \quad$ and 
$b_{\text {max }}^{2}=\left(\cosh ^{2}\left(D-\frac{1}{2}\right)\right)_{\text {max }} . \quad$ Now, $\quad c-\frac{b^{2}{ }_{\text {max }}}{\lambda_{1}}>0$ implies that $\pi^{2}\left(\frac{1}{X^{2}}+\frac{1}{D^{2}}\right)>3 \cosh ^{2}\left(D-\frac{1}{2}\right)$.

If $\frac{\pi^{2}}{D^{2}}>3 \cosh ^{2}\left(D-\frac{1}{2}\right)$ then the above will be satisfied for any $X>0$. This inequality in $D$ is satisfied if $D<D_{0} \approx 1.3$. Consequently flow is nonlinearly stable if the depth of the fluid layer $D$ is less than $D_{0} \approx 1.3$.

\section{Example 4}

We consider the example with the basic flow velocity $U_{0}(z)=\cosh z e^{z}$ and $b(z)=e^{2 z}$. Then the topography is a constant i.e., $T=2$ and $\quad \Psi^{\prime}(Q)=-\frac{1}{2} e^{4 z}<0 \quad$ where, $\quad c=\left(-\Psi^{\prime}(Q)\right)_{\min }=\frac{1}{2}$, $C=\left(-\Psi^{\prime}(Q)\right)_{\max }=\frac{e^{4 D}}{2}$ and $b_{\text {max }}^{2}=e^{4 D}$. So $c-\frac{b_{\text {max }}^{2}}{\lambda_{1}}>0$ will be satisfied if $\frac{\pi^{2}}{D^{2}}>e^{4 D}$ and this will be satisfied if $D$ lies in the range $0<D<0.63$.

\section{Concluding Remarks}

In this paper we have studied the nonlinear stability of steady basic flows in sea straits with variable cross sections with respect to finite amplitude disturbances by using the method of Arnol'd [1]. If $\Psi(x, z)$ is the stream function and $Q(x, z)$ is the vorticity divided by the width function $b(z)$ such that $\Psi=\Psi(Q)$, then our first stability theorem proves the nonlinear stability of basic flows satisfying the condition $\Psi^{\prime}(Q)>0$. Our second stability theorem ensures the nonlinear stability of basic flows satisfying the condition $\Psi^{\prime}(Q)<0$ but under the additional condition that the 
flow domain is sufficiently small. The above two theorems are valid for general steady flows. For the special case of plane parallel flows $\Psi=\Psi(z)$ and $Q=Q(z)$ we get the nonlinear stability of these flows when $Q(z)$ is a monotonic function of $z$. It is interesting to observe that the nonlinear stability conditions $Q^{\prime}(z)>0$ or $Q^{\prime}(z)<0$ are the same as the linear stability conditions obtained earlier in Deng et al [2]. Some examples of basic flows that are nonlinearly stable are also given.

It may be noted here that the linear stability of shear flows in sea straits has been developed when the fluid is homogeneous or stratified (cf. Deng et al [2], Subbiah and Ganesh [15]) but the nonlinear stability analysis developed in the present paper is restricted to homogeneous fluids only. A nonlinear stability analysis of stratified shear flows in sea straits by the use of the method of Arnol'd [1] may be taken up in the future.

\section{Acknowledgement}

V Ramakrishnareddy is thankful to the UGC for awarding a UGC-JRF.

\section{References}

[1] V I Arnol'd, On an a priori estimate in the theory of hydrodynamical stability, Amer. Math. Soc. Trans. Ser. 2, vol. 79, pp. 267-269, 1969.

[2] J Deng, L Pratt, L Howard and C Jones, On stratified shear flows in sea Straits of arbitrary cross section, Studies in Appl. Math., vol. 111, pp. 409-434, 2003.

[3] L J Pratt, H E Deese, S P Murray and W Johns, Continuous dynamical Modes in straits having arbitrary cross sections with applications to the Bab al Mandab, J. Phys. Oceanogr., vol. 30, pp. 2515-2534, 2000. 
[4] P G Drazin and W H Reid, Hydrodynamic Stability, Cambridge University Press, Cambridge, UK, 1981.

[5] M Subbiah and V Ganesh, On the stability of homogeneous shear flows in sea straits of arbitrary cross section, Indian J. Pur Appl. Math., vol. 38, pp. 43-50, 2007.

[6] M Subbiah and V Ganesh, On short wave stability and sufficient conditions for stability in the extended Rayleigh problem of hydrodynamic stability, Proc. Indian Acad. Sci. (Math Sci), vol. 120, pp. 387-394, 2010.

[7] M Subbiah and V Ramakrishnareddy, On the role of topography in the stability analysis of homogeneous shear flows, Journal of Analysis (to appear).

[8] D D Joseph, Stability of fluid motions I, II, Springer, 1976.

[9] V I Arnol'd, Conditions for nonlinear stability of stationary plane curvilinear flows of an ideal fluid, Sov. Math. Dokl., vol. 6, pp. 773-776, 1965.

[10] V I Arnol'd and B Khesin, Topological Methods in Hydrodynamics, Applied Mathematical Sciences, vol. 125, Springer, 1998.

[11] C Marchioro and M Pulvirenti, Mathematical theory of incompressible non-viscous fluids, Applied Mathematical Sciences, vol. 95, Springer, 1995.

[12] D D Holm, J E Marsden and T Ratiu, Nonlinear stability of the Kelvin-Stuart cat's eyes flow, Lect. in Appl. Mathematics, AMS, vol. 23, pp. 171-186, 1986.

[13] M E McIntyre and T G Shepherd, An exact local conservation theorem for finite-amplitude disturbances to non-parallel shear flows with remark on Hamiltonian structure and on Arnol'd's stability theorems, J. Fluid Mech., vol. 181, pp. 527-565, 1987.

[14] M Subbiah and M Padmini, Note on the nonlinear stability of equivalent barotropic flows, Indian. J. Pure Appl. Math., vol. 30, pp. 1261-1272, 1999. 
[15] M Subbiah and V Ganesh, Bounds on the phase speed and growth rate of the extended Taylor-Goldstein problem, Fluid Dynamics Research, vol. 40, pp. 364-377, 2008. 